\title{
A synopticity test of a sampling pattern in the Alboran Sea
}

\author{
Michel Rixen ${ }^{\mathrm{a}, *}$, Jean-Marie Beckers ${ }^{\mathrm{b}, 1}$ \\ ${ }^{a}$ Georges Deacon Division, Southampton Oceanography Centre, European Way, Southampton, S014 3ZH, UK \\ ${ }^{\mathrm{b}}$ GHER, B5 Institut de Physique, Sart Tilman, Université de Liège, 4000 Liège, Belgium
}

Received 10 January 2001; accepted 18 December 2001

\begin{abstract}
Hydrographic surveys are usually considered as synoptic, as if the corresponding data set had been sampled at the same time. However, this assumption might lead to strong biases in subsequent analyses. To obtain synoptic data at a given moment, we integrate a Lagrangian motion equation towards a 'modal sampling time', to relocate the sampling stations both in time and space, by combining objective analysis and geostrophic velocities, assumed to be either stationary or unstationary over the period of integration. The relocation algorithms are applied to a data set covering the entire Alboran Sea. The relocation of data points leads to a significant change on vertical velocities computed with the OMEGA equation. For validation purpose, the algorithm is also applied to a synthetic data set obtained from a 3D Primitive Equation (PE) model output. Raw and pseudosynoptic analysed temperature-salinity (T/S) fields and derived quasigeostrophic $(\mathrm{QG})$ vertical velocities are compared. It is shown that the relocation method might correct significantly the errors arising from the synopticity hypothesis and that the sensitivity to the level of no-motion and the analysis parameters is less important than the nonsynopticity. (C) 2002 Elsevier Science B.V. All rights reserved.
\end{abstract}

Keywords: Synopticity; Relocation; Alboran Sea; QG vertical velocities; 3D primitive equation model

\section{Introduction}

The aim of this paper is to assess the validity of the synopticity hypothesis of a particular data set covering the entire Alboran Sea and to validate simple relocation algorithms that partially correct the biases that arise from this hypothesis.

\footnotetext{
* Corresponding author. GHER, B5 Institut de Physique, Sart Tilman, Université de Liège, 4000 Liège, Belgium. Tel.: +32-43663650; fax: +32-4-3662355.

E-mail addresses: m.rixen@ulg.ac.be, myr@soc.soton.ac.uk (M. Rixen).

${ }^{1}$ Research Associate, National Fund for Scientific Research, Belgium
}

Hydrodynamic cruise data are usually considered as synoptic, as if the corresponding measurements had been sampled simultaneously. However, this assumption might lead to strong biases in temperaturesalinity $(\mathrm{T} / \mathrm{S})$ analyses or other variables that were measured and analysed (e.g. Wunsch, 1989; BohleCarbonell, 1992). In addition, variables that are then deduced from the original data may even be more sensitive to the analysis. An example is the calculation of the vertical velocity from the analysed T,S fields by means of the diagnostic OMEGA equation which is known to react strongly to changes in the data and in the analysis (e.g. Hoskins and Draghici, 1977; Hoskins et al., 1978; Tintoré et al., 1991; Pollard and Regier, 1992; Pinot et al., 1996; Viúdez et al., 1996; Allen and Smeed, 1996). 
Objective analysis (OA) (e.g. Gandin, 1965; Brasseur et al., 1996a) is very efficient when the database used is rich and redundant (e.g. Allen, 1974; Tchernia, 1977; Levitus, 1982; Brasseur et al., 1996a), but when cruise data are considered, a change in only one data point can produce a significant change in the analysis due to the relative small amount of data (e.g. Jones, 1972; Chelton, 1983; Bohle-Carbonell, 1992; Brankart, 1996). We can thus expect that a displacement (relocation) of the data can also modify the analysis. But since vertical velocity is a key factor for the biochemical exchanges in the water column (Woods, 1988; Pinot et al., 1996), particular attention should be paid to the vertical velocities computed from the T/S fields through the diagnostic OMEGA equation (e.g. Hoskins and Draghici, 1977; Hoskins et al., 1978).

Recent studies deal with this particular problem. It is first worthwhile to mention that the terminology 'quasi-synoptic' or 'nonsynoptic data set' is used in several publications (e.g. Golnaraghi et al., 1996; Viúdez et al., 1996), pointing out the fact that, apart from mapping errors arising from poor-resolution samplings, some error contributions from space/time smearing of the ocean dynamics are also expected in the analysis.

Matthews (1997) examines the impact of nonsynoptic sampling on mesoscale oceanographic surveys with towed instruments by using an isopycnal model: temporal changes in the model lead to a $40-50 \%$ increase in the RMS errors compared to those due to the spatial separation of the sampling points. Those errors result in a distortion of the mesoscale features and are unevenly distributed across the region of study.

Different analysis schemes may even behave differently when dealing with nonstationary oceanographic fields. Sokolov and Rintoul (1999) have compared the relative accuracy of optimal interpolation, spline interpolation, multiquadric-biharmonic interpolation and inverse distance weighting interpolation in the case of highly anisotropic processes and conclude that spline interpolation is a robust analysis scheme when applied to such kind of data.

Similarly, McGillicuddy et al. (2001) examine the synopticity of the US GLOBEC Georges Bank broadscale sampling pattern with observational system simulation experiments. Their approach uses model simulations that contain realistic spatial and temporal fluctuations of planktonic copepods to create a virtual cruise data set. The analysis of this synthetic data set indicates a total error of approximately $50 \%$ that is comprised mostly of simple mapping errors arising from the incomplete spatial sampling and a smaller contribution from the space/time smearing.

Analysis schemes (e.g. Bretherton et al., 1976; Brasseur, 1991) usually deal with any kind of data, and may be used in any spatial and/or temporal coordinate system if a suitable space and/or time correlation structure function is specified. This method, for example, has been used by Brankart and Pinardi (2001), where the weights on the data in a variational analysis scheme are modified according to the temporal window under study, and by Rixen et al. (2001b), where the weights are modified according to the sampling time with respect to a central reference sampling time.

Apart from these studies concentrating on the synopticity errors and sensitivity, several correction methods have been proposed recently. Perdersen et al. (2000), for example, use model velocities to obtain a synoptic sampling of Calanus finmarchicus by relocating the Lagrangian particles both in the $x y z$ and stage space. Their results indicate that the boundaries are more affected by their scheme whilst the general ecological interpretations are not dramatically changed. Gomis et al. (2000) use a linear interpolation between surveys to examine the synopticity of a particular data set by comparing raw and pseudosynoptic analysed fields and derived quasigeostrophic (QG) vertical velocities fields.

Allen et al. (2001) examine the errors in diagnostic vertical velocities and vertical temperature fluxes due to sampling strategies by using the output of an idealised numerical channel model with strong advection. The balance between number of observations and synopticity can lead typically to errors of $85 \%$ in the estimation of net vertical heat flux. In a companion paper (Rixen et al., 2001a), a simple relocation method is used to test the synopticity of the data set and is shown to correct the major part of the bias in hydrographic data when deriving vertical velocities.

Rixen et al. (2001b) investigate possible correction methods on the Omega data set, among which the same geostrophic relocation method and interpolation methods that are used in an assimilation experiment in a small area covering a part of the Atlantic Jet front in the Western Alboran Sea. In both last referred papers, the large advection imposes a wide relocation of water 
parcels and a significantly different distribution of the data, originally on a 'regular' grid. A major problem in these two examples of downstream sampling strategies is that whole areas are left void of data, thus increasing potentially the analysis errors.

For the purpose of the present study, dealing with these issues on other scales, we used a data set included in the MODB data base (Brasseur, 1995; Brasseur and Brankart, 1995; Brasseur et al., 1996b), originating from a field experiment carried out from 22 September to 7 October 1992 on board the R/V Garcia del Cid, resulting in 134 stations in a quasi-regular grid covering the entire Alboran Sea, a two basins area located between Spain and Morocco in the Mediterranean Sea. The distance between stations was usually $20^{\prime}$ longitude $(\sim 30 \mathrm{~km})$ and $10^{\prime}$ latitude $(\sim 18 \mathrm{~km})$. Vertical profiles were made down to $600 \mathrm{~m}$ except in coastal regions, with an interpolated accuracy of $1 \mathrm{~m}$ (Viúdez et al., 1996). Perkins et al. (1990) estimate typical length scales of $20-100 \mathrm{~km}$ in the Western Alboran Sea (WAS), whereas Gascard and Richez (1985) evaluate the internal Rossby radius in the Eastern Alboran Gyre (EAG) to $20 \mathrm{~km}$. More recently, in Allen et al. (1997), the Rossby radius is estimated to $15 \mathrm{~km}$. The horizontal spacing of the sampling appears to be subcritical compared to these physical space scales (i.e. not fine enough to retrieve all the mesoscale hydrodynamic features of the Alboran Sea). The short-term variability in the Western Alboran Basin (WAB) appears to be dominated by quasi-periodic variations in both strength and direction with a 5- to 9-day period (Perkins et al., 1990) with minima values down to 1 day. These time scales are shorter than the duration of the sampling. The assumption of synopticity is thus in doubt. Previous studies dealing with this data set actually never investigated that problem, despite the strong variability found in the Alboran Sea.

With respect to the previous studies, it is expected that the relocation will not leave entire areas void of data in this case. The displacement undergone by water parcels will probably be small with respect to the size of the area, even at the surface. Furthermore, stations might be trapped into one of both Alboran gyres, without the aggregation effect that was described by Rixen et al. (2001a,b) in the case of a strong advection.

The necessary assumptions of any relocation method should then be examined case by case, depending on the dynamics of the area under study, and verified/ validated by subsampling the state space of dynamical models (Bohle-Carbonell, 1992), which is the purpose of the present study.

This paper aims at investigating a possible bias in the data set arising from the lack of synopticity, at assessing the impact of this bias when deriving QG vertical velocities from the hydrographic field, at trying to correct this bias by applying relocation methods to the data, and overall, at validating the correction methods by using a synthetic data set obtained from a 3D Primitive Equation (PE) model of the area under study. We describe the methodology, the algorithms, the analysis and calibration tools in Section 2, the results of the relocation method on the raw data set in Section 3. In Section 4, the method is validated by means of a $3 \mathrm{D}$ PE model with the construction of a synthetic data set for which the 'true' solution is known. Finally, we draw some conclusions in Section 5.

\section{Methodology}

In this section, we will reconsider the relocation procedure, propose a slight modification to the stationary relocation version (Rixen et al., 2001a,b) and examine its applicability in the Alboran Sea.

When assuming that data have been collected simultaneously, we disregard the advection of the water parcels. However, the distance covered by a fluid particle during a given time interval is not necessarily related to the assumption of synopticity. The relevant terms are the local changes

$\frac{\partial T}{\partial t}, \quad \frac{\partial S}{\partial t}, \ldots$

but this information is not available from one single survey. Generally, we only have a series of measurements $i=1 \ldots N_{d}$ at locations $\boldsymbol{x}_{i}$ taken at instant $t_{i}$, with measurements of temperature $T_{i}$ and salinity $S_{i}$ (and eventually any other tracer $C_{i}$ ). If we assume a system without mixing, water parcels are moved around without changing their T,S characteristics. In a Lagrangian sense, we have

$\frac{\mathrm{d}\left[T_{i}, S_{i}, C_{i}\right]}{\mathrm{d} t}=0$

meaning $T_{i}, S_{i}$ and $C_{i}$ remain constant along trajectories. 
If we know the 3D velocity field $\boldsymbol{v}$ at each moment and location, we could retrieve the displacement of each data point by integrating

$\frac{\mathrm{d} \boldsymbol{x}_{i}}{\mathrm{~d} t}=\boldsymbol{v}(\boldsymbol{x}, t)$

where $\boldsymbol{v}$ is the $3 \mathrm{D}$ velocity.

To obtain water parcels trajectories and subsequently a synoptic data set at a given moment $t_{\text {ref }}$, it would be sufficient to integrate Eq. (3) from $t_{i}$ to $t_{\text {ref }}$ (which could be posterior or prior to $t_{i}$ ). Then classical OA could be used with the new positions and their corresponding data values $T_{i}, S_{i}$ or $C_{i}$. Unfortunately, we do not know the velocity fields (or at least we suppose the velocity field is not available), unless a hydrodynamic model is used or complex ADCP arrays are deployed, and no information on vertical velocities are available at this stage, since they usually remain below ADCP accuracy.

\subsection{The geostrophic equilibrium}

In the Alboran Sea, assuming typical values for the velocity field $(U=0.1 \mathrm{~m} / \mathrm{s})$, and the turbulent diffusivity $\left(\tilde{v}=10^{-2} \mathrm{~m}^{2} / \mathrm{s}\right)$ with a horizontal characteristic length $L_{\mathrm{h}}=\left[10^{4}-10^{5}\right] \mathrm{m}$ and a vertical characteristic length $L_{\mathrm{v}}=10^{2} \mathrm{~m}$, we note that the two adimensional numbers of Rossby and Ekman,

Ro $\sim 10^{-1}, \quad \mathrm{Ek} \sim 10^{-2}$

are small, except in frontal structures where the advection is relevant (i.e. the Rossby number might be high, approaching unity). Despite the particular frontal circulation in the Alboran Sea, the assumption of geostrophic equilibrium can thus be used to compute the velocity field and we will show that this hypothesis is suitable to gain accuracy in the diagnosed vertical velocity field.

Knowing the T/S fields, we are able to compute the geostrophic velocities. However, the integration of the thermal wind equation is subject to the choice of a reference level where the pressure field is known: this choice is not obvious, especially in the Mediterranean sea, where one can distinguish several water masses in the vertical (Tchernia, 1977; La Violette, 1994; Beckers et al., 1997). The vertical extension of the Western Alboran Gyre (WAG) is of the order of $200-220 \mathrm{~m}$ as shown by Viúdez et al. (1996), and they chose a reference level of 200 and $300 \mathrm{~m}$. This is not surprising, since the approximate level that separates eastward flowing Atlantic Water (AW) from westward flowing Mediterranean Water (MW) is found to be at $200-250 \mathrm{~m}$. So we may expect low velocities at these depths, at least for the special case of the Alboran Sea. To validate this first estimate of the level of no-motion, we used several measures $(\|\boldsymbol{u} \cdot \nabla T\|,\|\boldsymbol{u} \cdot \nabla S\|, \ldots)$, but none was able to elect uniquely one reference level. Furthermore, results were not very sensitive to the level of no-motion tested with values ranging between 200 and $300 \mathrm{~m}$.

\subsection{Relocation algorithm}

We have to choose a time $t_{\text {ref }}$ to which all data have to be relocated. The aim of the relocation method is thus to obtain a pseudosynoptic data set at time $t_{\text {ref. }}$ To limit average integration time, we compute a modal sampling time $t_{\text {ref }}=\left(t_{1}+t_{N_{d}}\right) / 2$. The choice of the reference sampling time $t_{\text {ref }}$ should be considered with care. Relocation to the beginning or the end of a survey is dangerous. Indeed, by increasing the integration time, one would increase the length of the trajectory of each station and the number of data points leaving the domain. Statistically, relocating to the middle of the survey will on average lead to shorter displacements and fewer mixing during advection (which is neglected in our method). To analyse both stationary and unstationary methods, this choice appeared to be particularly convenient to allow consistent comparisons over the whole Alboran Sea.

Data sampled before $t_{\text {ref }}$ will be downstreamed with the velocity field, whereas data past $t_{\text {ref }}$ will be upstreamed. In the case of a sampling following the main current, one expects the relocation to globally concentrate data points whilst in the case of a sampling against the main current, one expects the relocation to spread out data points.

The geostrophic assumption is a simple and easy way to estimate the velocity field from the T/S fields. In this case, the vertical velocity is zero, and data are only moved on their initial standard levels. So we assume that we only know the horizontal geostrophic velocity $\boldsymbol{u}$, moving the points according to

$\frac{\mathrm{d} \boldsymbol{x}_{i}}{\mathrm{~d} t}=\boldsymbol{u}(\boldsymbol{x}, t)$ 
The main problem that arises here is the time dependency of the velocity field. To integrate Eq. (5), we need the velocity field on the whole time interval covering the cruise. This could be computed if we knew the density field at each moment. This leads to two different methods (see Fig. 1).

The stationary method aims at calculating a single geostrophic field that is used to relocate the data points from the measurement location upstream or downstream according to the moment at which the measure was taken. Once points have been moved towards their expected synoptic location, the analysis is performed and the corresponding geostrophic velocity computed. If these new geostrophic velocity and density fields differ significantly from the previous one, the new geostrophic velocity is used to restart the procedure. After convergence, one obtains a geostrophic velocity field that advects water parcels in such a way that the analysis of the relocated data produces a density field that drives the geostrophic velocity $\boldsymbol{u}(\boldsymbol{x})$.

The unstationary method has the same objectives as the stationary method, but instead of using a single geostrophic velocity field during the whole cruise length, a series of $N$ fields are used at equidistant moments spread over the cruise time interval. The same iterative method is used to compute these differ- ent geostrophic currents, which are then interpolated linearly in time between the $N$ fields to obtain $\boldsymbol{u}(\boldsymbol{x}, t)$.

The first analysis (see Fig. 1, step 1) in both the stationary and unstationary methods is performed on the raw data set. Subsequent analyses are obtained from the relocated cruise stations. In the same manner, the initial velocity field (step 2) is computed from the raw data set and the subsequent geostrophic velocities are computed from the relocated analysed T/S fields.

Both methods are then self-consistent from the T/S data only, since no velocity information is needed.

\subsection{The variational inverse model}

To analyse the data, we used a variational inverse method (VIM) (Brankart and Brasseur, 1996, 1998; Rixen et al., 2001c), already widely tested on the Mediterranean (Brasseur et al., 1996b). It can be shown that in a statistical sense, the classical optimal interpolation scheme (OI) (see, e.g., Gandin, 1965; Bretherton et al., 1976) and the variational principle are equivalent (see Brasseur et al., 1996a; Brankart and Brasseur, 1996). The nondimensional formulation leads to a simple calibration through a characteristic length $L$ and a signal/noise ratio $S / N$.

The analysis method appeared to be relatively sensitive to both parameters, and they have thus to

- Stationary method: the geostrophic velocity is constant in time during the entire relocation. In this case, the procedure which leads to an adjusted density-velocity field is:

(1) T,S analysis with raw data stations at each standard level

(2) geostrophic velocity computation $\boldsymbol{u}_{\text {ref }}$ from $\mathrm{T} / \mathrm{S}$ analysis

(3) relocation of data stations from $t_{i}$ to $t_{r e f}$ using geostrophic velocities $\boldsymbol{u}_{r e f}$

(4) T,S analysis with relocated data stations at each standard level

(5) repeat steps 2 to 4 until convergence

- Unstationary method: the velocity is time dependent. In this case, the procedure which leads to an adjusted density-velocity field is:

(1) T,S analysis with raw data stations at each standard level

(2) geostrophic velocity computation $\boldsymbol{u}_{\text {ref }}$ from $\mathrm{T} / \mathrm{S}$ analysis used as initial $\boldsymbol{u}_{r e f_{k}}$

(3) For $N$ values $t_{r e f_{k}}$ in $\left[t_{1} . . t_{N_{d}}\right]$

- relocation of data stations from $t_{i}$ to $t_{r e f_{k}}$ using the time interpolated geostrophic velocities $\boldsymbol{u}_{r e f_{k}}$

- $T_{k}, S_{k}$ analysis with relocated data stations at each standard level

- geostrophic velocity computation $\boldsymbol{u}_{r e f_{k}}$ at $t_{k}$ using $T_{k}, S_{k}$

(4) repeat step 3 until convergence of relocated field at $t_{r e f}$

Fig. 1. Stationary and unstationary relocation algorithms. 
be optimised to minimise the statistical analysis error, to correctly filter out the noise of the data and to extract the proper information contained in the data set. Indeed, a systematic bias in the analysis would compromise the relocation procedure. By smoothing the field too much for example, the Western Alboran Gyre could collapse. One possible way to obtain the optimal statistical parameters $L$ and $S / N$ is to compute an approximation of the covariance function from close but nonoverlapping data, to fit these results to an a priori known correlation function and to extrapolate this function at the origin (e.g., Brankart and Brasseur, 1996). This is indicated for climatological data, when disposing a large amount of overlapping data. A first investigation showed that the use of this simple method to evaluate the statistical properties of a (relocated) data set was insufficient in the case of a single cruise.

Recently, Brankart and Brasseur (1996) have shown that the generalised cross validation (GCV) (Craven and Wahba, 1979; Golub et al., 1979; Wahba, 1990) may be a suitable and efficient way to extract from the database itself the optimal interpolation parameters by applying the analysis tool on several data sets from which some data were eliminated. Calculating the difference between the analyses on the full and the restricted data set provides an estimation of the quality of the analysis. The set of optimal calibration parameters is then the one that minimises this statistical error estimator.

The generalised cross validation method has been applied to the raw data set and we used these optimal statistical parameters $(L$ and $S / N)$ for the analysis needed during the relocation procedure. We could imagine to cross-validate the relocated data set to compute the relocated fields. This would lead to an iterative procedure, unless the statistical features of the relocated field are not modified anymore. At this stage, this method has not been implemented yet, and appears to be quite expensive. In any case, we will verify that reasonable changes in $L$ and $S / N$ produce only small changes in the raw analysed fields, whereas the relocation actually modifies significantly the analysis.

Any analysis needs some a priori information about the background field (e.g. Gandin, 1965; Brasseur, 1991). It is the best prior estimate of the target field and the choice of this field is preliminary to any analysis. Usually, it is chosen as the mean or the linear regression of the data. For highly varying field, this can lead to systematic errors in the solution (Brankart and Brasseur, 1996). For reasons that will be detailed later, this solution is also not close enough to reality in regions void of data. An alternative proposed by (Brankart and Brasseur, 1996) is to compute the reference field from a semi-normed spline analysis, leaving out the underived term in the smoothing operator. Provided the background field is smooth enough, it will not significantly influence the solution.

The numerical resolution of the variational principle is based on a finite element method and the corresponding mesh used was constructed from a simplified contour of the Alboran Sea with a characteristic length of $0.05^{\circ}(\sim 4.4 \mathrm{~km})$ according to our first estimation of the characteristic length of both $T$ and $\mathrm{S}$ fields. This length is not critical since each finite element is divided into three subelements each one modelling a polynomial of order 3 . Finer meshes were tested but did not show significant gain in precision. We chose to extend the mesh outside of the sampling area, because we might expect relocation of data outside of the domain.

\subsection{Vertical velocities}

Estimation of vertical velocity is a key factor for the exchanges of heat and salt between the surface and the ocean interior. It is also responsible for the vertical transport and distribution of nutrients, plankton,... (e.g. Woods, 1988; Nihoul et al., 1989; Pinot et al., 1996).

As direct measures of vertical velocities are difficult, because they usually remain below ADCP accuracy, several indirect methods have been used. Strass (1994) has compared three different tools to evaluate them, among which the diagnostic OMEGA equation, which he showed to give the best estimates of vertical velocity. Different formulations of the OMEGA equation can be found. The classical one is known as quasigeostrophic (QG) (e.g. Hoskins et al., 1978).

To take higher order dynamics into account, Hoskins and Draghici (1977) established a semigeostrophic (SG) OMEGA equation. The latter includes ageostrophic advection, which was neglected in the QG formulation. The diagnostic equation in this case can be obtained by a coordinate transformation. 
Pinot et al. (1996) showed that the QG method can lead to systematic biases because it relies on assumptions that do not properly represent the ageostrophic frontal dynamics. Indeed, the QG method does not adequately take into account horizontal ageostrophic advection. In this paper, the vertical velocity fields were computed with the QG OMEGA formulation, since no structural differences were found between the QG and the SG methods. Differences appeared only in the magnitude of upwelling and downwelling velocities $(\sim 5-10 \%)$.

To solve the QG equations, boundary conditions are needed. The most trivial and first idea is to set null vertical velocities $(w=0)$ at the surface, at the bottom and at lateral boundaries. Although it might be obvious to use this assumption at the surface, the justification for bottom and lateral conditions is not straightforward. While horizontal velocities, the forcing of the OMEGA equation, and therefore $w$ are usually much weaker at $200 \mathrm{~m}$ than those observed at 100 m (Viúdez et al., 1996), the absence of topographic effect is not straightforward in the Alboran Sea. In Viúdez et al. (1996), to validate their model, a flat bottom domain of $200 \mathrm{~m}$ depth was used, thus avoiding topographic artifacts in their study. The choice of a null vertical velocity gradient $(\partial w /$ $\partial z=0$ ) did not change significantly the vertical velocity field (Viúdez et al., 1996). Hence, null vertical velocity boundary conditions were used in all our computations.

\section{Results}

\subsection{Raw data set}

The analysis parameters are calibrated by the GCV. The optimal characteristic length between the surface and $300 \mathrm{~m}$ for both $\mathrm{T} / \mathrm{S}$ fields ranges between 10 and $60 \mathrm{~km}$. The optimal $\mathrm{S} / \mathrm{N}$ ratio ranges between 1 and 100 for $T$ and between 10 and 1000 for $S$. The standard deviation of the background field error for $T$ ranges between $1.7{ }^{\circ} \mathrm{C}$ at the surface and $0.05{ }^{\circ} \mathrm{C}$ at $300 \mathrm{~m}$ and for $S$ between 0.5 at the surface and 0.02 at $300 \mathrm{~m}$. The standard deviation of the observational error for $T$ ranges between $0.5{ }^{\circ} \mathrm{C}$ at the surface and $0.02{ }^{\circ} \mathrm{C}$ at $300 \mathrm{~m}$ and for $S$ between 0.5 at the surface and 0.002 at $300 \mathrm{~m}$. It is worth noting that the sensitivity of the analysis to $S / N$ and $L$ are both of the same order of magnitude. This was already mentioned by Brankart (1996) in the case of both a small database and quasi-synoptic data, as opposed to climatological data sets, where the characteristic length is not a sensitive parameter.

Based on the small Rossby radius of deformation, the analysis is finally interpolated on a $0.05^{\circ} \times 0.05^{\circ}$ grid, similar to the finite element mesh characteristic length, to avoid accidentally smoothing the field. In the vertical, 15 equally spaced levels were considered from 20 to $300 \mathrm{~m}$ (one level every $20 \mathrm{~m}$ ).

The temperature and geostrophic velocity field at $100 \mathrm{~m}$ are shown in Fig. 2. The level of no-motion is set at $240 \mathrm{~m}$. The raw data set clearly exhibits two anticyclonic gyres, the WAG and the Eastern Alboran Gyre (EAG), together with other small mesoscale features. The Atlantic jet initiating the Almeria-Oran front is also clearly visible at $100 \mathrm{~m}$ for both fields. There are small cyclonic gyres southwest of the EAG and west of the WAG. The signature of both gyres is, however, less clear at the surface, due to the perturbations of atmospheric processes. These analyses are in good agreement with the results obtained by Viúdez et al. (1996) who use an analysis method based on a successive correction iteration (Pedder, 1993).

The QG vertical velocity field computed from the raw data set is shown in Fig. 3. The distribution of upwelling and downwelling region is closely related to ridges and throughs found in the T,S and density field. Maximum significant values are about 5-7 m/ day with a strong upwelling upstream of the WAG and a strong downwelling downstream of the WAG.

\subsection{Relocated data set}

The relocation algorithm has been applied here to the real raw data set. Convergence, in the sense of the relocation algorithm that was defined in the previous section (i.e. mutual adjustment between density and geostrophic velocity field) was fairly well obtained after a few tens of iterations. We note (see Fig. 4, top) that after relocation, the sampling points have been moved horizontally towards the middle of the domain, although several stations are trapped and recirculated in either of the two gyres. The displacements are shorter at deeper levels because geostrophic velocities decrease with depth. The trajectories of the stations 


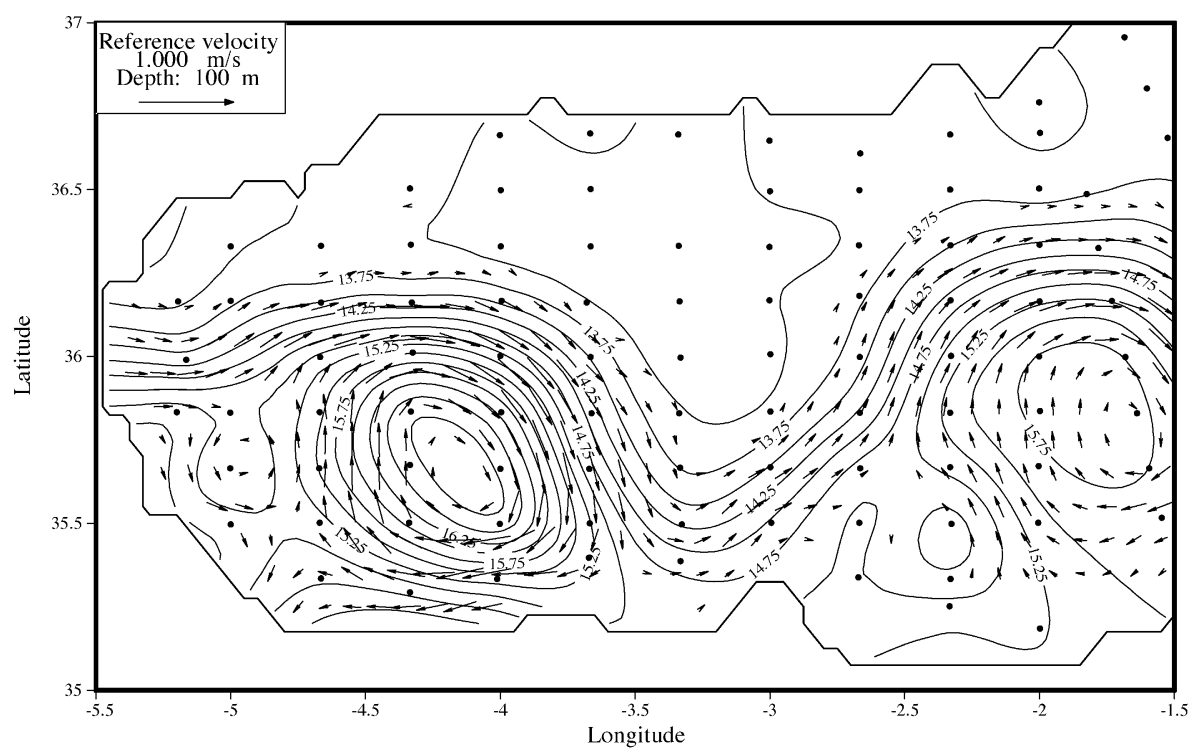

Fig. 2. Temperature $\left({ }^{\circ} \mathrm{C}\right)$ and geostrophic velocity field obtained from the raw data set at $100 \mathrm{~m}$.

for the unstationary relocation method (Fig. 4, bottom) look very similar, but overlap slightly, indicating the nonstationary behaviour of the currents in the Alboran Sea. The data points that are relocated outside of the domain are not used in the subsequent analyses.
At a first glance, the analysed fields computed from the relocated T,S data sets remain almost unchanged (see Fig. 5 for temperature): the major Alboran Sea features (WAG, EGA,...) are conserved after relocation However, at $100 \mathrm{~m}$ depth, the strength of the T,S front and the magnitude of geostrophic velocities have

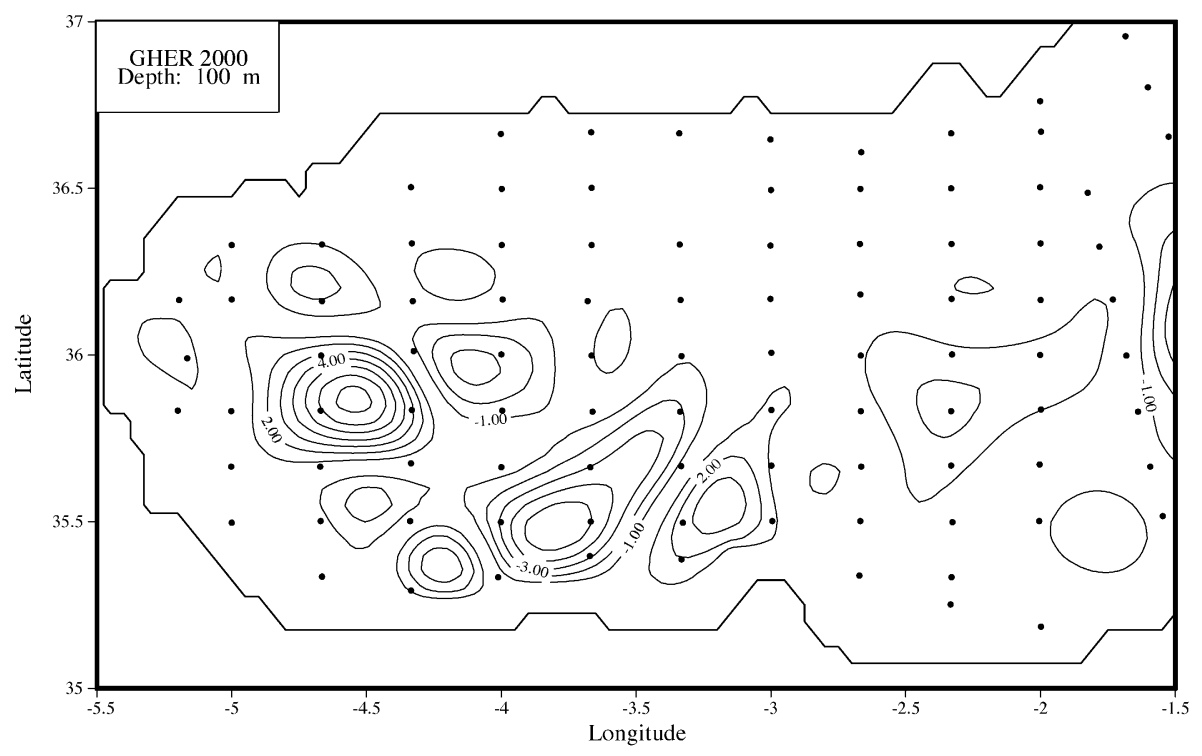

Fig. 3. QG vertical velocities $\left(10^{-5} \mathrm{~m} / \mathrm{s}\right)$ at $100 \mathrm{~m}$ computed from the raw data set. 

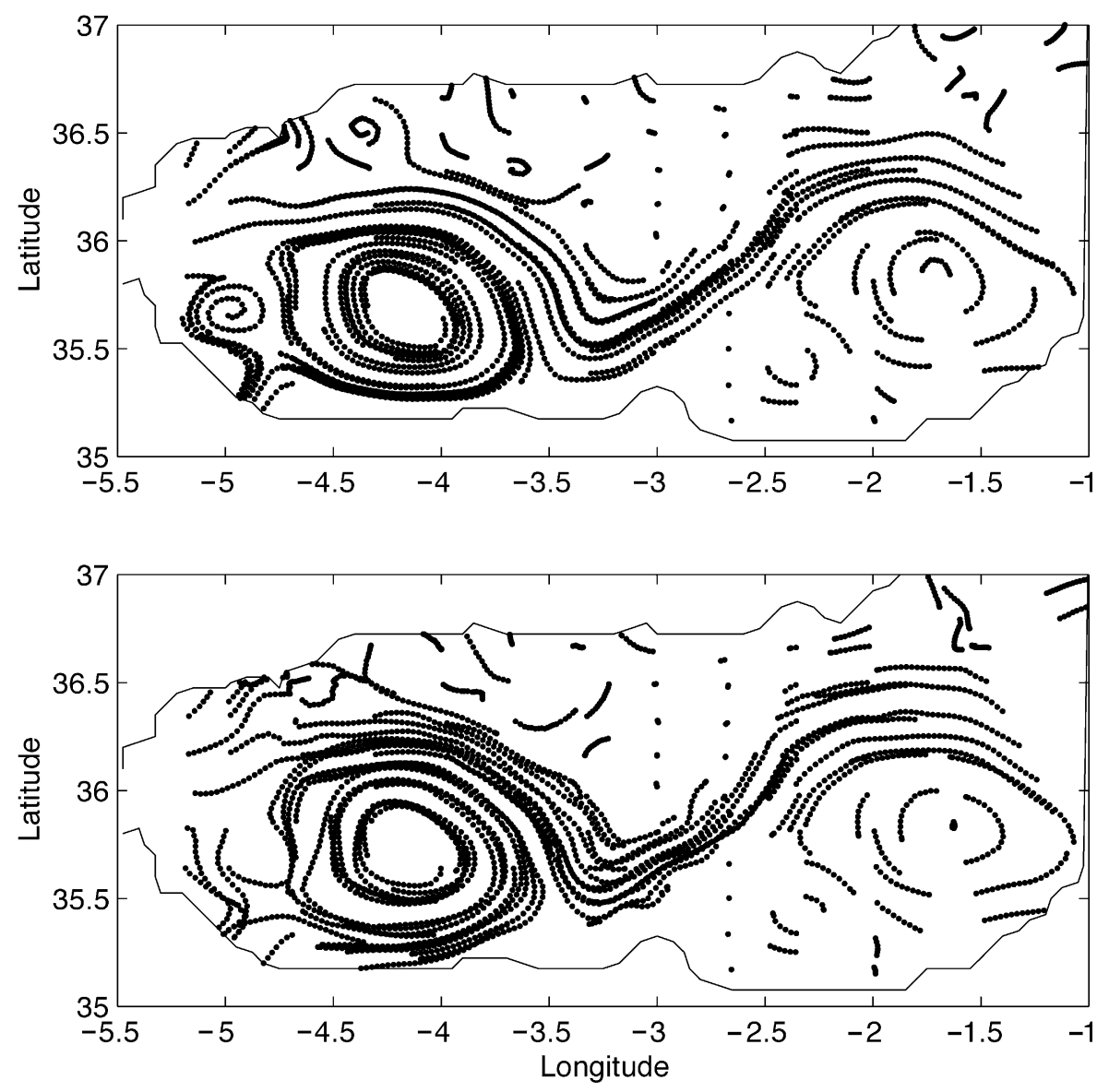

Fig. 4. Relocation trajectories at $20 \mathrm{~m}$ of the raw data stations obtained from the stationary (top) and unstationary (bottom) methods.

been slightly increased. A small cyclonic structure west of the WAG has disappeared, because the area is now void of data.

The QG vertical velocities obtained from the relocated data set are quite different (see Figs. 6 and 7) from the ones obtained from the raw data set analysis (Fig. 3). The magnitude of upward/downward motion cells has been increased (about 10-40\%) as expected, especially in the WAG area, as a result of the relocation of a downstream sampling pattern, which concentrates water parcels and thus increases horizontal gradients (Rixen et al., 2001a). The effect is stronger for the unstationary method. Although the changes in the T,S fields are tiny, the integration of the OMEGA equation shows that derived vertical velocities are strongly sensitive to synopticity errors and hence to the relocation.
One may wonder where the changes in the vertical velocities come from. First, it should be noted that the QG vertical velocities are more sensitive to the relocation than the T,S fields, since vertical velocities are obtained from the density field second derivatives. Moreover, the relocation implies a relative horizontal displacement of the stations. This modifies the forcing of the OMEGA equation through the $\boldsymbol{Q}$-vector. Indeed, the OMEGA forcing is computed from a combination of geostrophic shear and curvature and the relocation may directly act on one or both of these characteristics.

The relocation procedure transforms the original regular grid of experimental data points into a set of irregular locations, which might be an important source of errors in the analysed fields (e.g. Jones, 1972; Bohle-Carbonell, 1992). Objective analysis 


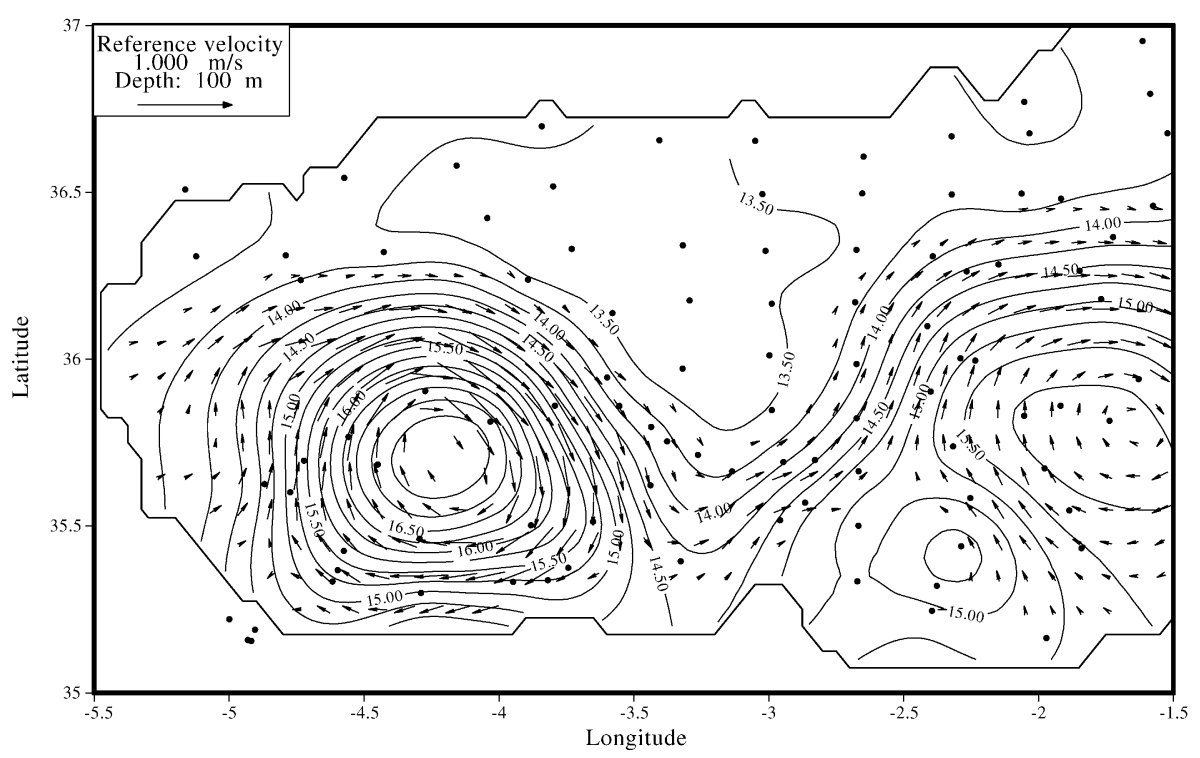

Fig. 5. Temperature $\left({ }^{\circ} \mathrm{C}\right)$ and geostrophic velocity field at $100 \mathrm{~m}$ obtained from the relocated raw data set (stationary method).

does, however, not make any assumption about the regularity of the data distribution and the synopticity bias could actually be solved by providing a suitable space/time correlation function. This can be done by applying a modified weight to the data, according to the lapse between $t_{\text {ref }}$ and the time the data were sampled (see, e.g., Rixen et al., 2001b). In the method used here, the correlation function is purely spatial and the analysis is time independent. Instead, some dynamic information is used to modify the data position.

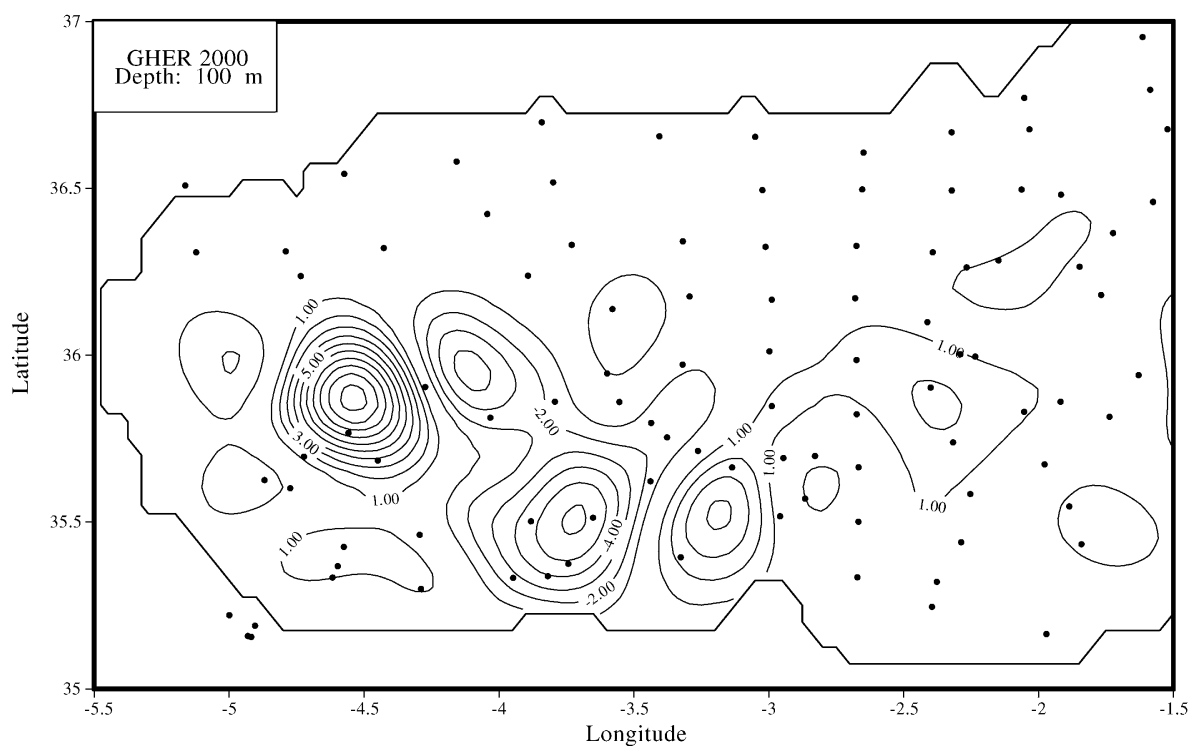

Fig. 6. QG vertical velocities $\left(10^{-5} \mathrm{~m} / \mathrm{s}\right)$ at $100 \mathrm{~m}$ computed from the relocated raw data set (stationary method). 


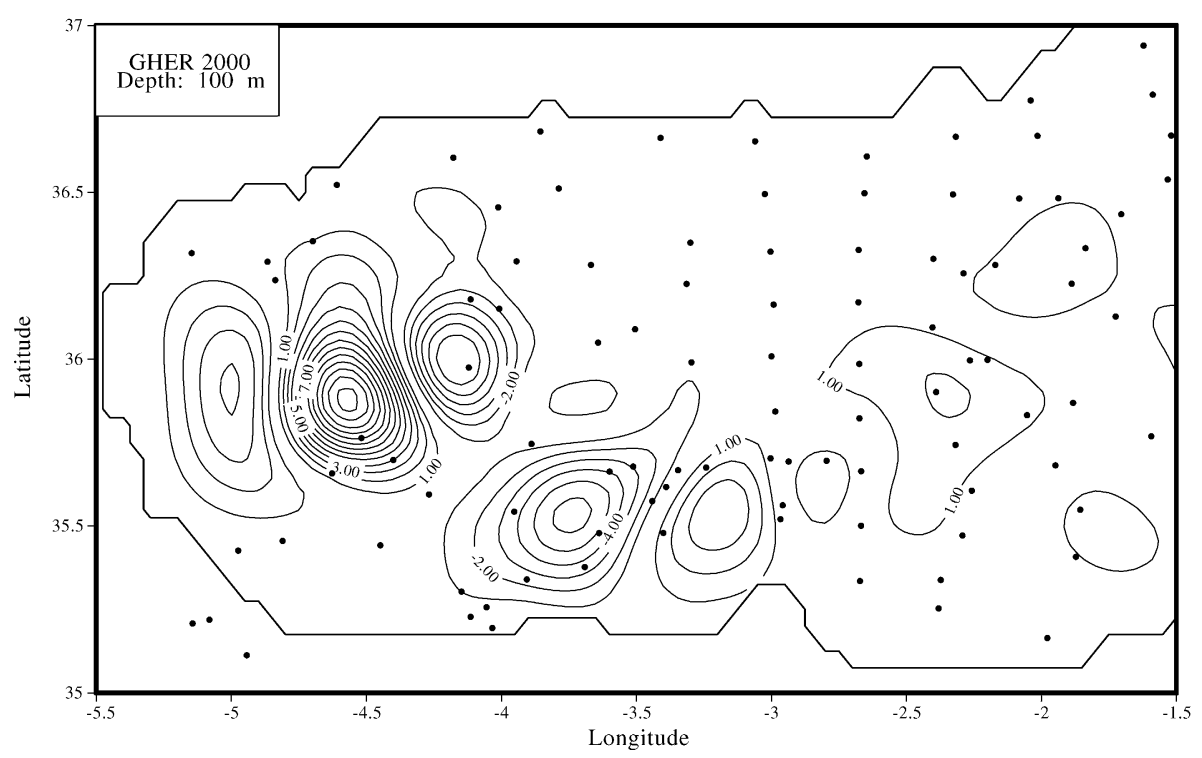

Fig. 7. QG vertical velocities $\left(10^{-5} \mathrm{~m} / \mathrm{s}\right)$ at $100 \mathrm{~m}$ computed from the relocated raw data set (unstationary method).

The fact that data points are lost as a consequence of the relocation procedure might be seen as a serious pitfall of the method, but the recirculation of water parcels avoids the definitive lost of information in the frontal regions chosen. Furthermore, there is some redundant information in the raw data set, since the cruise was performed downstream, measuring several times the same water mass, and the loss of information is thus not worrying. Finally, the reference field keeps a trace of the original raw data set information in areas void of data.

Slight differences appear in the T,S fields and the geostrophic velocity field when changing the reference level respectively to 200 and $300 \mathrm{~m}$, or when using suboptimal statistical parameters (changing the $S / N$ by a factor 10 and the correlation length by a factor 2). These small changes are cumulated during the relocation, resulting in dissimilarities in the station positions. Despite the low sensitivity of geostrophic velocities on the reference level, the level of nomotion plays an important part in the relocation, since it may directly modify the velocity field and furthermore the displacement undergone by the data. The sensitivity to the reference level and the analysis were found to be less than the sensitivity to the relocation method, both for the stationary and the unstationary relocation methods. The geostrophic approximation is thus valid at these space and time scales and allows us to test the relocation method. The same conclusions apply to the QG vertical velocities.

\section{Validation}

The previous section showed that the relocation method may modify both magnitude and position of the upward/downward motion cells. But how may we know which of the raw or relocated data set is closer to reality? We need at this stage an objective method to assess the relative quality of these analyses and vertical velocities. By means of an already spun up 3D PE model, run over about 15 days, we developed the following twin experiment (see Fig. 8 ). First, we extracted the cruise from the consecutive model results with the spatio-temporal references of the real cruise data used in the previous section. The extraction is done at each vertical level used for the analysis. This provided a 'synthetic' raw data set. Secondly, we relocated the 'synthetic' raw data set with the same algorithm as used for the real data. Finally, we compared both synthetic raw and relocated analyses to the actual $3 \mathrm{D}$ output of the 


\section{Model T,S}

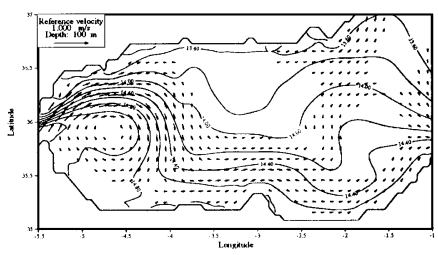

Extract synthetic survey
Model QG $w$ at $t_{r e f}$
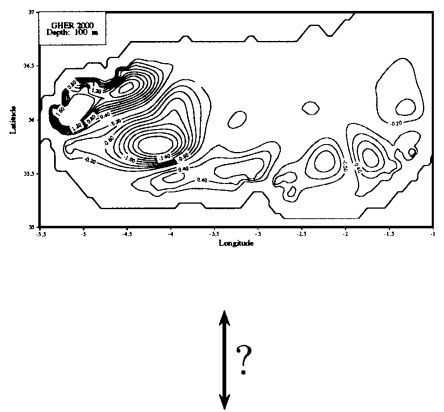

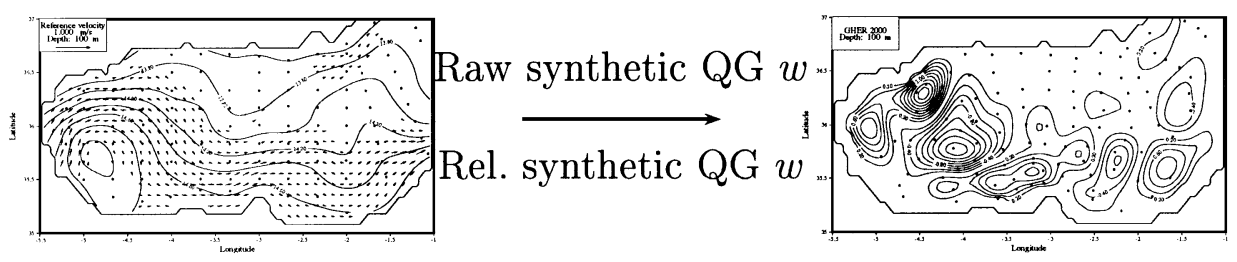

Fig. 8. Schematic representation of the twin experiment validation. A survey is extracted from the results of the 3D PE model and the raw and relocated data sets QG vertical velocities are compared to the QG $w$ output of the model at $t_{\text {ref. }}$.

model with specific control state variable: T/S, geostrophic velocities and specially the QG vertical velocities.

\subsection{The model}

The present implementation is based on the GHER mathematical model described, for example, in Nihoul et al. (1989) and Beckers (1991).

As was already mentioned by Tintoré et al. (1991), vertical velocities due to mesoscale phenomena are an order of magnitude larger than the vertical motion associated with the large-scale gyres. Thus, the choice of the grid size must correctly fit mesoscale processes. From the characteristic length of the data and the internal radius value (see Section 1), the grid has to be at least as small as $5 \mathrm{~km}\left(\sim 0.05^{\circ}\right)$. The $3 \mathrm{D}$-model domain extends from $-9.5^{\circ}$ to $-0.815^{\circ}$ in longitude, and from $33.36^{\circ}$ to $37.5^{\circ}$ in latitude, both in the Atlantic Ocean and in the Alboran Sea. The application of the model has effectively shown that it is easier to impose open-sea boundary conditions far from the
Strait of Gibraltar. This allows a slow propagation of the hydrodynamic conditions through the strait. Thus, the above-mentioned resolution leads to a 3D $193 \times$ $117 \times 31$ matrix.

Both $\mathrm{T} / \mathrm{S}$ fields are initialised with the MODB climatology (September conditions). For stability reasons, we imposed a relaxation to the MODB climatology at the open-sea boundary conditions with a characteristic time of 2 months. The initial velocity field and the surface elevation are obtained from the geostrophic approximation. The initial turbulent kinetic energy $k$ field is solved from the stationary $k$ equation.

We impose zero vertical scalar fluxes and a logarithmic profile for the velocity (classical turbulent friction layer) at bottom and lateral boundaries. For air-sea boundary conditions, a monthly averaged climatological wind stress was used to compute vertical fluxes for temperature and salinity. Zero normal gradient are imposed on open-sea boundaries for $\mathrm{T}, \mathrm{S}$, $k$, normal, tangential and mean tangential velocity on both sides of the domain. 


\subsection{The model output}

The output of the model at $t_{\text {ref }}$ (see Fig. 9) clearly exhibits the WAG. The absence of the eastern gyre might be explained by the monthly averaged atmospheric forcing (a finer-daily - forcing was not yet available). It is also known that this gyre is not permanent (e.g., Viúdez et al., 1996).

The results produced by the model do not show small mesoscale features like the raw data set did. However, this fact is not relevant since the $3 \mathrm{D}$ model is only needed here to provide a consistent raw data set and a 'synoptic' vertical velocity field to which our raw and relocated fields will be compared to. The comparison, after spinup, of the velocity fields of the model and the geostrophic velocities obtained from the $\mathrm{T} / \mathrm{S}$ output of the model leads to the following result: at the surface, the geostrophic velocity represents about $80 \%$ of the total velocity in the particular region chosen. Thus, although the ageostrophic component is known to be important in the Alboran Sea (topographic effects, Atlantic jet,...), it appears worthwhile to test the relocation method, since the geostrophic approximation is reasonably well satisfied.

The model contains higher order dynamics that the QG OMEGA formulation cannot reproduce with the boundary conditions chosen. Thus, comparing diagnosed vertical velocities to the output of the 3D- model does not make sense. Consequently, all the vertical velocities will be compared to the $\mathrm{QG}$ model vertical velocities computed from the OMEGA equation (see Fig. 10), considered as the 'true' reference solution of our twin experiment. The two important upwelling and downwelling cells are located as expected, respectively upstream and downstream of the WAG with maximum vertical velocity magnitude of $2 \mathrm{~m} /$ day.

\subsection{Synthetic raw data set}

The synthetic raw data set analysis (see $\mathrm{T}$ field on Fig. 11) has been obtained by extracting the cruise from the consecutive model outputs, since each measurement is characterised by a spatio-temporal value. The analysed T/S fields are quite different compared to the model output, indicating a significant variability in the model that the (synthetic) survey is not able to retrieve entirely, nor in time, nor in space: This is yet another proof of the coarseness of the cruise which cannot reproduce all the hydrodynamic output from the model. The WAG as reproduced by the model is in a slightly growing phase. As the survey was planned downstream (i.e. eastward), the sampling of the WAG occurs before $t_{\text {ref, }}$, thus underestimating both the size and the strength of the gyre, also partially lost by the low spatial resolution of the sampling. The changes in

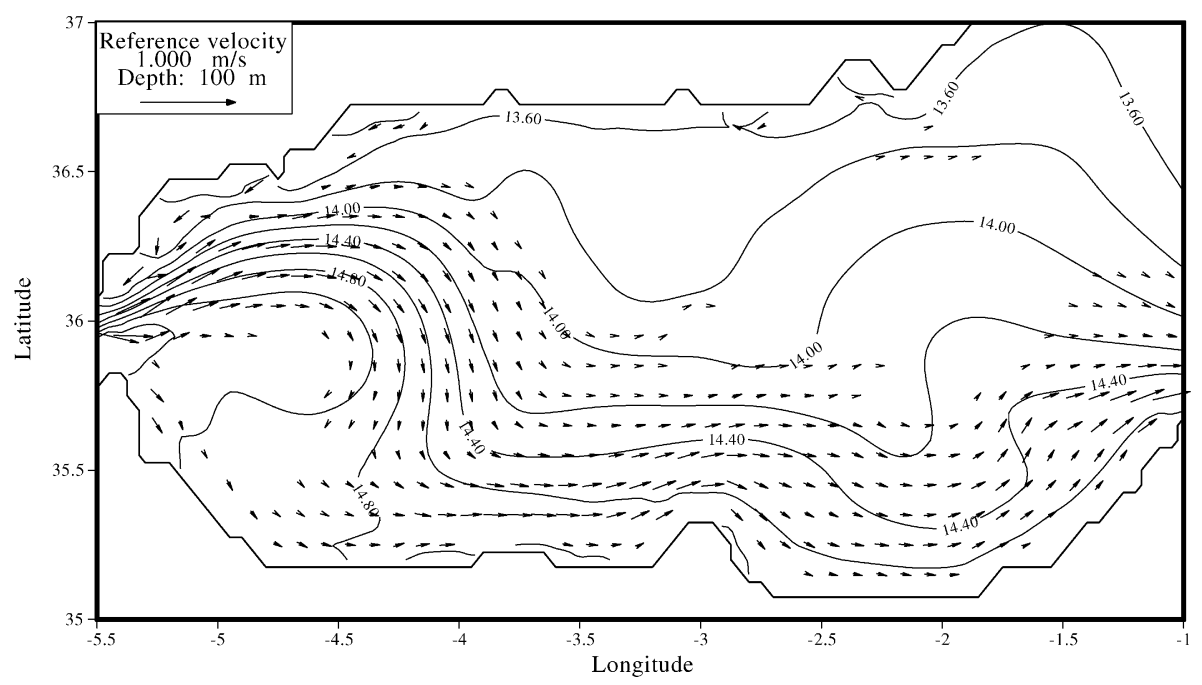

Fig. 9. Model temperature $\left({ }^{\circ} \mathrm{C}\right)$ and geostrophic velocity field at $100 \mathrm{~m}$. 


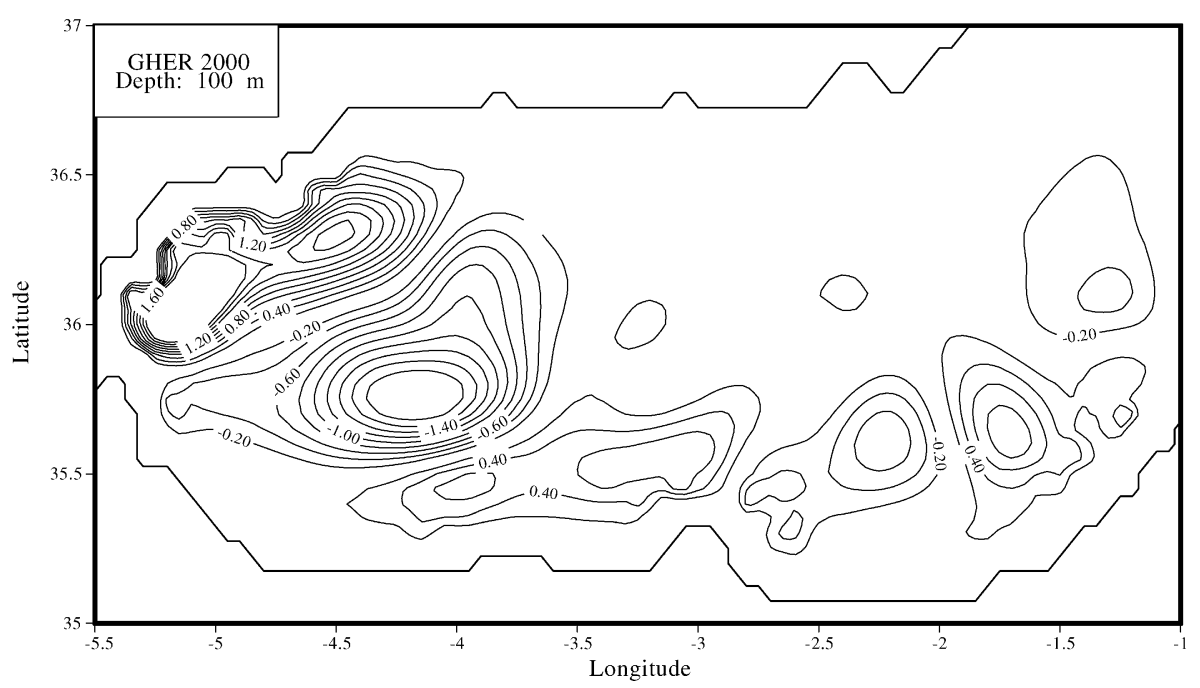

Fig. 10. QG vertical velocity $\left(10^{-5} \mathrm{~m} / \mathrm{s}\right)$ at $100 \mathrm{~m}$ obtained from the $\mathrm{T} / \mathrm{S}$ output of the model.

the T/S fields are reflected in the QG vertical velocities computed from the synthetic raw data set (see Fig. 12). We note that in the Western Alboran Basin (resp. Eastern Alboran Basin), the magnitude of QG vertical motion is globally less (resp. higher) than the ones obtained from the model, because the survey is sampling a growing wave whose strength is underestimated at the beginning of the cruise and overestimated at the end of the cruise.

\subsection{Synthetic relocated data set}

As expected (see Fig. 13), due to the organisation of the cruise, the stations are relocated horizontally towards the middle of the Alboran Sea, between the WAG and the EAG, thus leaving some regions without data at the western and eastern boundaries of the Alboran Sea. Compared to the analysis of the synthetic raw data set (see Fig. 11), the relocated analysis

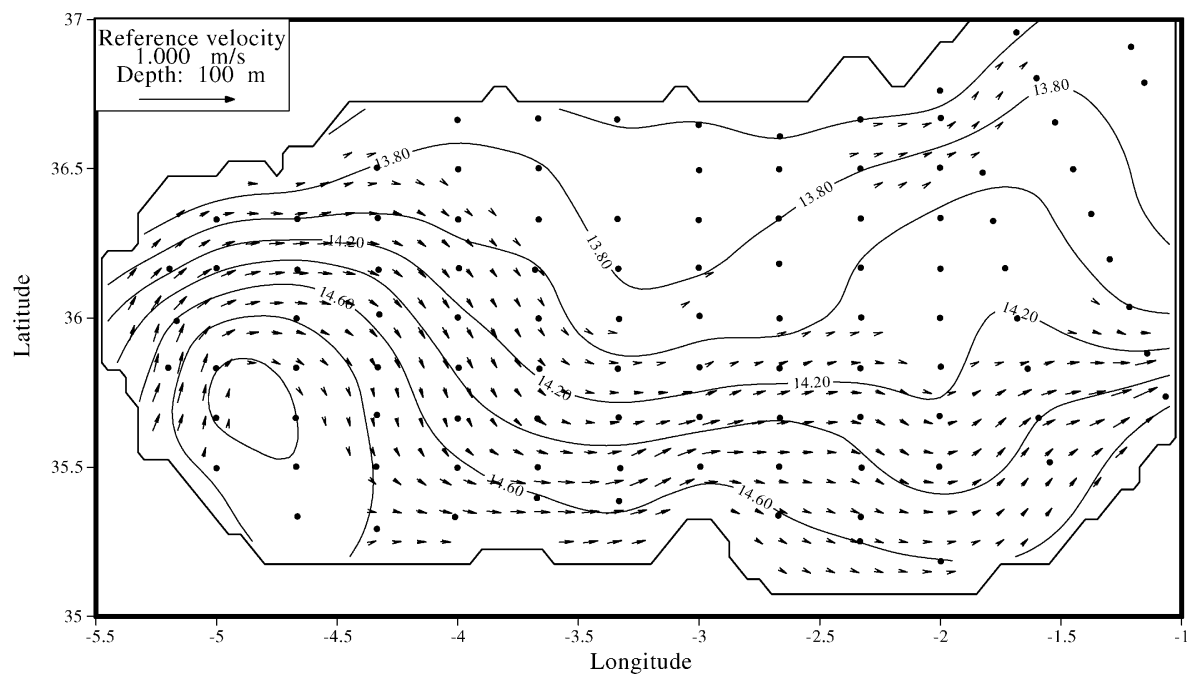

Fig. 11. Temperature $\left({ }^{\circ} \mathrm{C}\right)$ and geostrophic velocity field at $100 \mathrm{~m}$ obtained from the synthetic raw data set. 


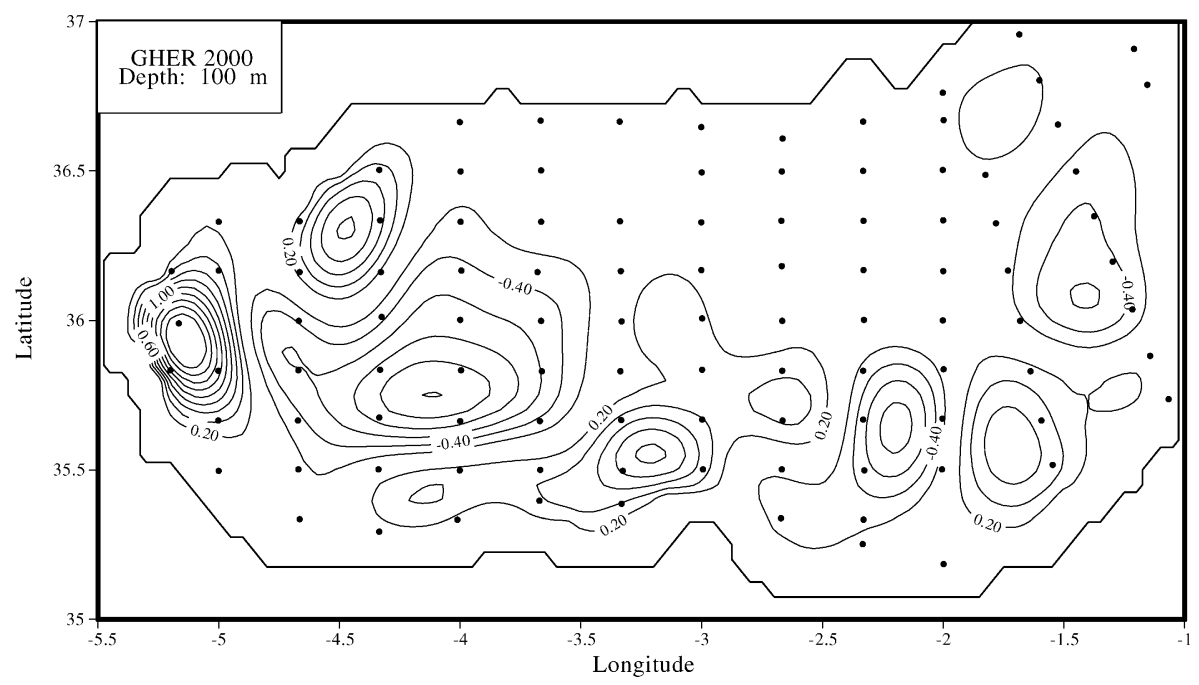

Fig. 12. QG vertical velocity $\left(10^{-5} \mathrm{~m} / \mathrm{s}\right)$ at $100 \mathrm{~m}$ obtained from the synthetic raw data set.

shows a stronger front downstream of the WAG, whereas upstream, the area has been left void of data. The size of the WAG has also been increased, according to the $\mathrm{T}$ model output at $t_{\text {ref. }}$ The hydrographic fields obtained by using the unstationary method provided similar results: at a first glance, no significant difference was noted. The mean separation between the stations of both relocation methods was less than $7 \mathrm{~km}$.
While the T,S and the geostrophic velocity fields are only slightly changed, as in the case of the real cruise data, it is interesting to lay emphasis on the following question: does the relocation (and its geostrophic hypothesis) increase the accuracy of the vertical velocity field, or is this hypothesis damping the ageostrophic hydrodynamic processes?

Examining Fig. 14, for data covered areas, qualitatively, we note a better correspondence between

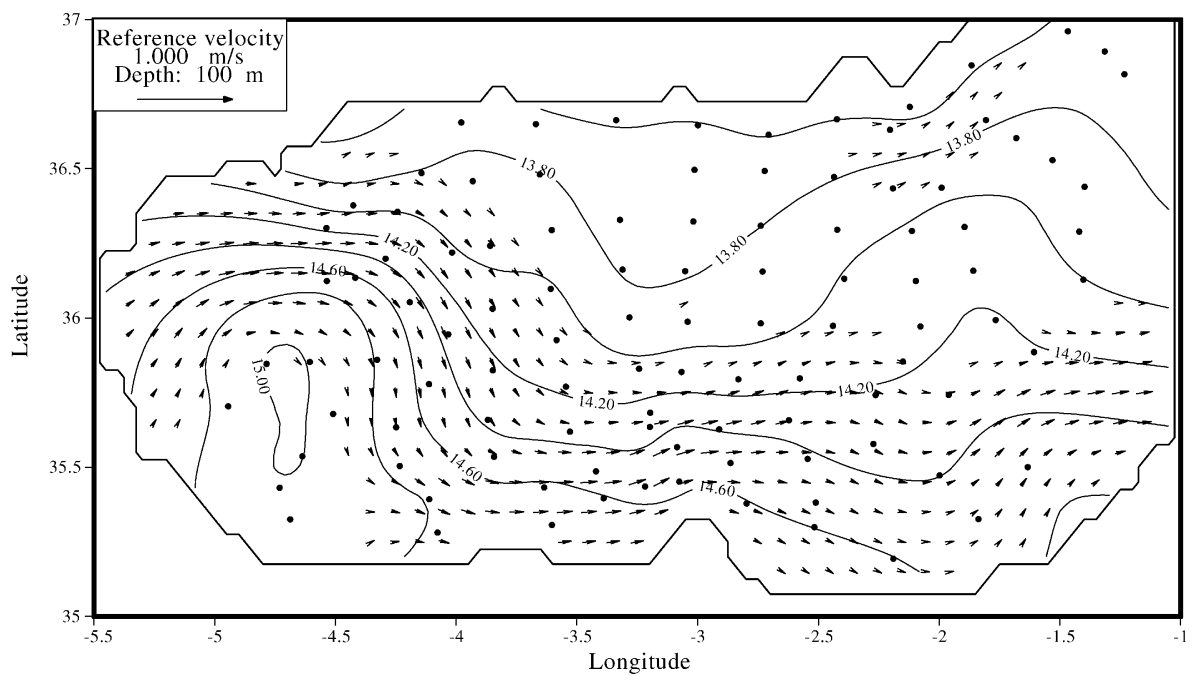

Fig. 13. Temperature $\left({ }^{\circ} \mathrm{C}\right)$ and geostrophic velocity field at $100 \mathrm{~m}$ obtained from the relocated synthetic raw data set (stationary method). 


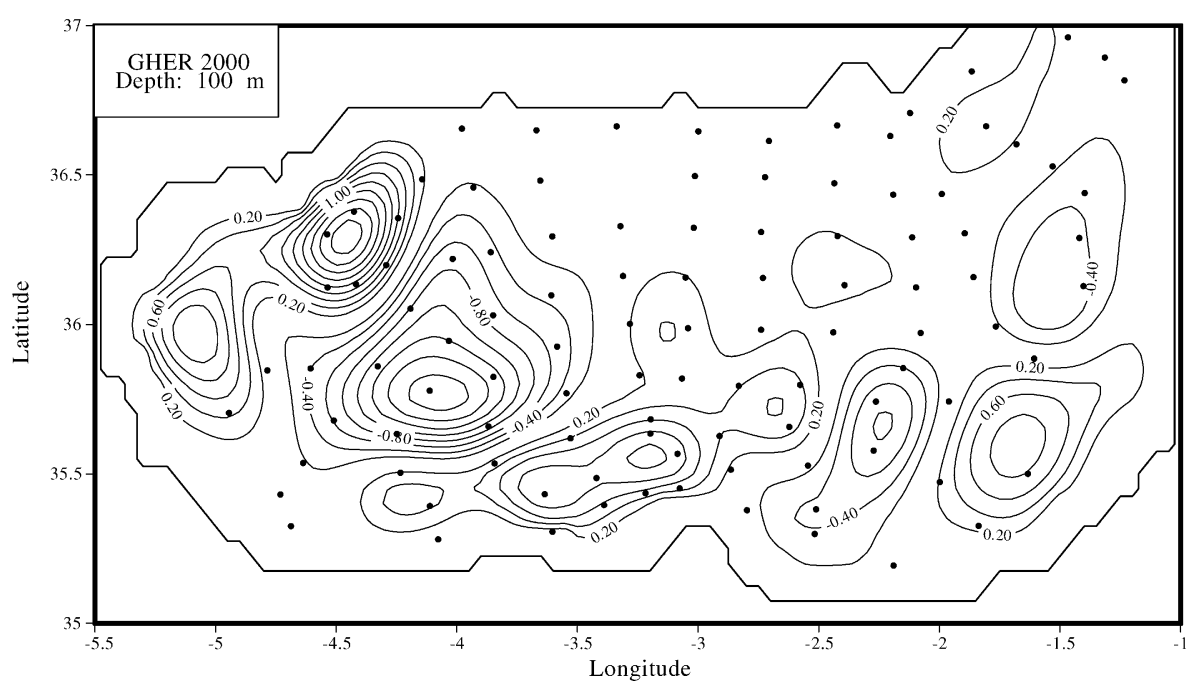

Fig. 14. QG vertical velocity $\left(10^{-5} \mathrm{~m} / \mathrm{s}\right)$ at $100 \mathrm{~m}$ obtained from the relocated synthetic data set (stationary method).

pseudosynoptic QG vertical velocity magnitude and the QG model vertical velocities: there is a general increase of $w$ after relocation, due to the increase of gradients in the hydrographic fields resulting from the relocation. Conclusions for the unstationary relocation are similar again (Fig. 15). There is, however, a significant difference between both fields at the western edge of the WAG, an area void of data.

We must keep in mind that the sampling itself induces a loss of information. Indeed, the sampling represents only a part of a complete field information, depending on the sampling resolution. By sampling the synoptic model result with the initial and relocated grid, we obtain analyses that take into account this statistical effect. The latter field gives a kind of 'best solution' that could be obtained from a relocation procedure with the relocated grid, since this synthetic sampling gives the 'exact' values of T,S at these points.

Quantitatively (see Table 1), we measured RMS values and the correlations on vertical velocities

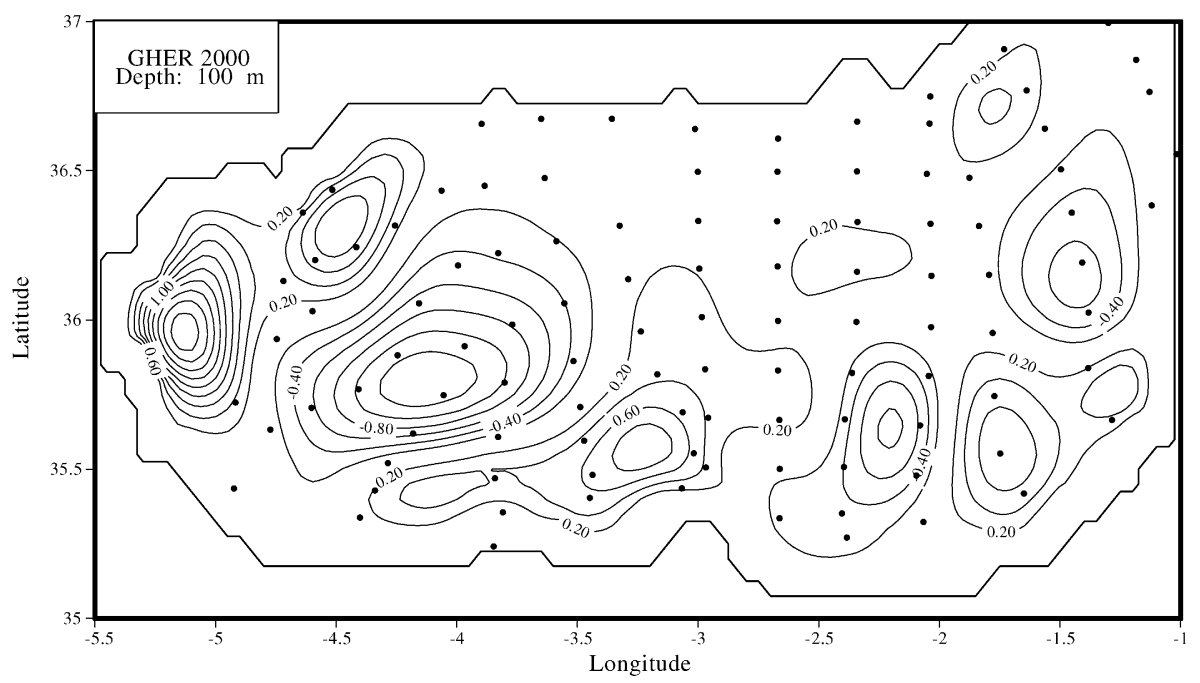

Fig. 15. QG vertical velocity $\left(10^{-5} \mathrm{~m} / \mathrm{s}\right)$ at $100 \mathrm{~m}$ obtained from the relocated synthetic data set (unstationary method). 
Table1

RMS $\left(10^{-5} \mathrm{~m} / \mathrm{s}\right)$ and correlation on QG vertical velocities at $100 \mathrm{~m}$ between the model QG output (the reference) and the QG vertical velocities obtained from (1) the model output reconstructed with the initial grid, (2) the model output reconstructed with the relocated grid ('best' value obtainable with the given sampling), (3) the synthetic raw data set, (4) the synthetic raw data set (reference level at $200 \mathrm{~m}$ ), (5) the synthetic raw data set (reference level at $300 \mathrm{~m}$ ), (6) the synthetic raw data set (smoothed analyses), (7) the synthetic raw data set (noised analyses), (8) the relocated synthetic raw data set (stationary relocation), (9) the relocated synthetic raw data set (unstationary relocation), (10) the relocated synthetic raw data set (unstationary relocation, reference level at $200 \mathrm{~m}$ ), (11) the relocated synthetic raw data set (unstationary relocation, reference level at $300 \mathrm{~m}$ ), (12) the relocated synthetic raw data set (unstationary relocation, smoothed analyses), (13) the relocated synthetic raw data set (unstationary relocation, noised analyses)

\begin{tabular}{lll}
\hline Method & RMS & Correlation \\
\hline 1. modts.valid (S) & 0.27 & 0.87 \\
2. modts.stat.valid (S) & 0.28 & 0.86 \\
3. valid (NS) & 0.37 & 0.73 \\
4. valid.200 (NS) & 0.37 & 0.73 \\
5. valid.300 (NS) & 0.38 & 0.72 \\
6. valid.smooth (NS) & 0.38 & 0.72 \\
7. valid.noisy (NS) & 0.38 & 0.72 \\
8. stat.valid (PS) & 0.34 & 0.78 \\
9. unstat.valid (PS) & 0.34 & 0.78 \\
10. unstat.valid.200 (PS) & 0.35 & 0.77 \\
11. unstat.valid.300 (PS) & 0.35 & 0.77 \\
12. unstat.valid.smooth (PS) & 0.35 & 0.77 \\
13. unstat.valid.noisy (PS) & 0.35 & 0.77 \\
\hline
\end{tabular}

(S), (NS) and (PS) stand, respectively, for 'synoptic', 'non-synoptic' and 'pseudo-synoptic'.

between the QG model output on the one hand and several other raw and relocated fields on the other hand.

The QG model output (the reference for comparison, Fig. 10) refers to the vertical velocity field computed directly from the T/S model output relative to the 'modal sampling time'. The initial (line 1 of Table 1) and relocated (line 2) sampled model results refer to the model output sampled with initial and relocated cruise stations. This gives an order of magnitude of the loss of information on correlation due to the spatial sampling (13\% for the initial grid and $14 \%$ for the relocated grid). The latter value (line 2) gives a kind of upper bound of both RMS and correlation on synoptic data that could be obtained with the relocated grid. Subsequent results refer respectively to the QG vertical velocities for raw synthetic data (lines 3-7) and the relocated synthetic data (lines 8-13).
Comparisons of lines 1 and 2 reflect the general statement that a uniform complete spatial coverage gains better results than a non-uniform one if the field is synoptic. The error on synopticity (line 3 ) induces another loss of correlation up to $13 \%$ compared to line 1 . The cumulated sampling and synopticity error is about $27 \%$. It is also shown (lines $4-7$ ) that the use of other reference levels $(200$ or $300 \mathrm{~m})$ or the use of suboptimal statistical parameters $(L$ and $S / N$ changed by a factor 2 and 10, respectively) does not change significantly the results on the synthetic raw data. Moreover, the stationary and unstationary relocation (lines 8 and 9) show the improvement on QG vertical velocities, both on RMS and correlation. In absolute terms, the correlation has been raised from $73 \%$ to $78 \%$. But in relative terms to the model synoptic sampling (lines 1 and 2), the correlation has been raised from $84 \%$ to $91 \%$. The sensitivity study (lines $10-13)$ shows that the unstationary relocation is not sensitive neither to changes in the level of no-motion nor to changes in the statistical parameters of the analysis. The same similar sensitivity study applied the stationary method provides similar conclusions.

Some comments on the unstationary relocation are needed here. The nonstationarity $\left|\partial \boldsymbol{u}_{\boldsymbol{g}} / \partial t\right|$ of the raw geostrophic fields (see previous section) is more important than the synthetic geostrophic fields ones by a factor $\sim 5$. Hence, the model does not fully represent the whole variability of the hydrodynamic processes found in the real data. The RMS between the overall model geostrophic velocity on the one hand and the corresponding stationary and unstationary geostrophic velocities $u$ and $v$ components of $\overrightarrow{\boldsymbol{u}}_{\boldsymbol{g}}$ on the other hand do not show significant differences: $u$ is better by $0.4 \%$ for the unstationary method whilst $v$ is better for the stationary method by $1.5 \%$.

As a sequence of geostrophic velocities is used, the solution is more accurate than the stationary relocation method in the Western Alboran basin for a relocation at the beginning of the time period. The correlation and RMS in the Western Alboran sea at that time yield respectively 0.80 and 0.32 (on the data covered area) but over the whole Alboran Sea at the same time only 0.71 and 0.38 . Similarly, when relocating towards the end of the sampling period, it is slightly more accurate in the Eastern Alboran Sea. At that time on this area, the correlation and RMS values are respectively 0.79 and 0.34 whilst over the whole area only 0.73 and 
0.36 . The unstationary relocation of water parcels over the whole period using the time-dependent geostrophic velocities, however, does not seem to benefit from these local improvements.

In both cases, some areas are left without any data, because of the long relocation time and trajectories. Hence, the choice of the modal reference sampling time is fully justified. By construction, the unstationary method uses the velocity information where and when it is accurate, but the stationary and unstationary methods give, however, similar results and they do not show significant differences in sensitivity.

A GCV has been performed on the final stationary and unstationary relocated data sets, and shows that the characteristic length is roughly stable (changes of $-10 \%$ to $+10 \%$ ), but that the observational noise is usually lower by approximately $5-15 \%$.

In all cases, the relocation methods reduce the error significantly, and always in a more important way than changes in the level of no-motion or in the analysis parameters. The improvement expected from the unstationary relocation method is, however, not significant in this case.

\section{Conclusions}

The aim of this study was to develop a simple firstorder correction method to obtain pseudo-synoptic data sets from a raw data set by relocating data points with geostrophic velocities computed from the data set itself.

This study has led to several interesting results and conclusions. The synopticity of a particular cruise in the Alboran Sea was examined with regard to time and space scales. The sampling was found to be subcritical, thus not fine enough to retrieve all the information of the mesoscale activity in this region. A validation through a 3D hydrodynamic model proved objectively the correction obtained on the QG vertical velocity field by relocating the data in space and time. The basic hypothesis of geostrophy used in the relocation strategy is satisfactory enough in this case to correct to a first order the diagnostic QG vertical velocity on real data sets. It was also found that the QG omega vertical velocities were more sensitive to the relocation than to the reference level or the analysis.
To summarise, in this test case, when deriving QG diagnostic vertical velocities from cruise data, some $13 \%$ might be lost from the insufficient sampling resolution and some $14 \%$ might be lost from the nonsynopticity. On the contrary, neither the level of nomotion nor the analysis parameters seem to be determinant in the accuracy of the derived vertical motion. It should be also noted that the validation procedure used here is based on a 3D PE model with little temporal variability. With more realistic models, the synopticity of a cruise would probably be even more critical. Whether the validation procedure itself is valid is then strongly related to the representativity skills of the model. The limitation of this exercise probably lies in the low temporal variability observed in the model, driven only by forcings at low temporal resolution. The design of optimal sampling strategies via Observation System Simulation Experiments should be based indeed upon operational forecasting models.

The stationary and unstationary methods show similar results on QG vertical velocities (same RMS and correlation over the whole area) and similar sensitivity to the reference level and the analysis parameters. The related geostrophic velocities components are not systematically better for the unstationary relocation compared to the stationary relocation. These conclusions also apply to the hydrographic fields. The stationary relocation method under these spatio-temporal scales is then equivalent to the unstationary method and should hence be preferred as it is less expensive.

Some potential uses of the method are not only the improvement of analysis and their derived fields like vertical velocities, but also the possibility to plan cruises. Indeed, if some climatic hydrological fields are available, the method can be inverted so as to compute the sampling strategy which, after relocation, leads to a data grid that is optimised for the purpose of the study. The relocation method can also be used for a systematic sensitivity analysis when performing objective analysis on cruise data. Rather than to displace sample locations randomly around their original position to establish the sensitivity to the sampling strategy, the relocation method leads to a physically interpretable sensitivity.

At this stage, only T,S tracers were tested. As the relocation only depends on T/S analyses, the relocated stations can be used to compute relocated analyses for any passive tracer $\mathrm{C}_{i}$. 
The method may be, however, criticised on some points. If the relocation has such a clear effect on the vertical velocity field, does it not simply mean that the cruise planning was not done correctly? The remark supposes that one knows very well the feature that is going to be measured and that the physical components are those that impose the structure on the cruise. Also logistical impossibilities may not allow to modify the speed at which samples are taken.

Another possible problem is that the relocation may move points near the boundary into the domain, leaving the border regions void of data. This indeed happens and leads to an extrapolation in these regions. The problem of the extrapolation can, as shown, be dealt with by using an appropriate background field that guides the analysis in its extrapolation. Finally, one may wonder if there is not an inconsistency between relocating by a geostrophic velocity, while diagnosing vertical velocities from a semi- or quasigeostrophic approach. The point here is that solving the OMEGA equation without relocation is even worse and the relocation is simply a first-order correction consistent with the standard QG approach using geostrophic velocities to approximate nonlinear terms.

\section{Acknowledgements}

This work would not have been possible without the help and expertise of J.-M. Pinot and D. Gomis (UIB, Spain). We thank P. Brasseur (IMG, Grenoble, France), J.-M. Brankart (IMGA, Bologna, Italy), R. Haney (NPS, Monterey, USA), J. Allen (SOC, Southampton, UK) and two anonymous reviewers for their precious comments and suggestions. Support for this study was obtained through E.C. MASTIII programme (project OMEGA MAS3-CT95-0001). The data set used originates from the MODB (MASTII programme, project MAS2 CT93.0075.BE) data base. The development of the 3D PE model of the GHER (GeoHydrodynamics and Environment Research) was financed during several years by the European Commission (EROS2000, EUROMODEL, MEDMEX, MODB/IS, OMEGA, MATER and TOROS projects). IBM Belgium, IBM Europe and IBM International Foundation provided supercomputer assistance, most recently in the scope of the ERP-
SALMON project (Sea Air Land Modelling Operational Network). The first author currently benefits from an EC Marie Curie fellowship under programme "Energy, environment and sustainable development" (contract EVK2-CT2000-50004).

\section{References}

Allen, D., 1974. The relationship between variable selection and data augmentation and a method for prediction. Technometrics $16,125-127$.

Allen, J.T., Smeed, D., 1996. Potential vorticity and vertical velocity at the Iceland-Faeroes front. J. Phys. Oceanogr. 26 (12), 2611 2634.

Allen, J.T., Smeed, D., Crisp, N., Ruiz, S., Watts, S., Velez, P., Jornet, P., Rius, O., Castellón, A., 1997. Upper ocean underway operations on BIO Hesperides cruise OMEGA-ALGERS (cruise 36) using SeaSoar and ADCP 30/09/96-14/10/96. Technical Report no. 17, Southampton Oceanography Center, 29 pp.

Allen, J.T., Smeed, D., Nurser, A., Zhang, J., Rixen, M., 2001. Diagnosing vertical velocities using the QG Omega equation: an examination of the errors due to sampling strategy. Deep-Sea Res. 48 (2), 315-346.

Beckers, J.-M., 1991. Application of a 3D model to the Western Mediterranean. J. Mar. Syst. 1, 315-332.

Beckers, J.-M., Brasseur, P., Nihoul, J.C.J., 1997. Circulation of the western Mediterranean: from global to regional scales. DeepSea Res. 44 (3-4), 531-549.

Bohle-Carbonell, M., 1992. Pitfalls in sampling, comments on reliability and suggestions for simulations. Cont. Shelf Res. 12 (1), $3-24$.

Brankart, J.-M., 1996. Modélisation statistique de l'hydrologie méditerranéenne. Validation et contrôle de qualité d'une climatologie de référence. $\mathrm{PhD}$ thesis, University of Liège, Collection des publications, Sciences appliquées, 209 pp.

Brankart, J.-M., Brasseur, P., 1996. Optimal analysis of in situ data in the Western Mediterranean using statistics and cross-validation. J. Atmos. Oceanic Technol. 13 (2), 477-491.

Brankart, J.-M., Brasseur, P., 1998. The general circulation in the Mediterranean Sea: a climatological approach. J. Mar. Syst. 18, 41-70.

Brankart, J.-M., Pinardi, N., 2001. Abrupt cooling of the Mediterranean Levantine Intermediate Water at the beginning of the 1980s: observational evidence and model simulation. J. Phys. Oceanogr. 31, 2307-2320.

Brasseur, P., 1991. A variational inverse method for the reconstruction of general circulation fields in the Northern Bering Sea. J. Geophys. Res. 96 (C3), 4891-4907.

Brasseur, P., 1995. Free data offered to researchers studying the Mediterranean. Eos Trans. 76 (37), 363.

Brasseur, P., Brankart, J.-M., 1995. The Mediterranean oceanic data base. MAST Days Proceedings. EEC Publication.

Brasseur, P., Beckers, J.-M., Brankart, J.-M., Schoenauen, R., 1996a. Seasonal temperature and salinity fields in the Mediter- 
ranean Sea: climatological analyses of an historical data set. Deep-Sea Res. 43 (2), 159-192.

Brasseur, P., Brankart, J.-M., Beckers, J.-M., Rixen, M., $1996 \mathrm{~b}$. Seasonal Variability of Hydrodynamical Fields in the Mediterranean. University of Liège, GHER, Liège, Belgium, 253 pp.

Bretherton, F.P., Davis, R.E., Fandry, C.B., 1976. A technique for objective analysis and design of oceanographic experiment applied to MODE-73. Deep-Sea Res. 23, 559-582.

Chelton, D., 1983. Effects of sampling errors in statistical estimation. Deep-Sea Res. 30, 1083-1103.

Craven, P., Wahba, G., 1979. Smoothing noisy data with spline functions: estimating the correct degree of smoothing by the method of generalized cross-validation. Numer. Math. 31, $377-403$.

Gandin, L.S., 1965. Objective Analysis of Meteorological Fields. Israel Program for Scientific Translation, Jerusalem, $242 \mathrm{pp}$.

Gascard, J., Richez, C., 1985. Water masses and circulation in the Western Alboran sea and in the Strait of Gibraltar. Prog. Oceanogr. $15,157-216$

Golnaraghi, M., Lozano, C., Robinson, A., 1996. Dynamical adjustment of quasi-synoptic data sets in the Eastern Mediterranean: testing a full basin coastal methodology. J. Mar. Syst. 7, 9-42.

Golub, G.H., Heath, M., Wahba, G., 1979. Generalized cross-validation as a method for choosing a good ridge parameter. Technometrics 21 (2), 215-223.

Gomis, D., Ruiz, S., Pedder, M., 2000. Diagnostic analysis of the $3 \mathrm{D}$ ageostrophic circulation from a multivariate spatial interpolation of CTD and ADCP data. Deep-Sea Res. 48 (1), 269-295.

Hoskins, B.J., Draghici, I., 1977. The forcing of ageostrophic motion according to the semigeostrophic equations and in an isentropic coordinate model. J. Atmos. Sci. 34, 1859-1867.

Hoskins, B.J., Draghici, I., Davies, H., 1978. A new look at the omega equation. Q. J. R. Meteorol. Soc. 104, 31-38.

Jones, R., 1972. Aliasing with unequally spaced observations. J. Appl. Meteorol. 6, 245-254.

La Violette, P.E., 1994. Seasonal and Interannual Variability of the Western Mediterranean Sea. Coastal and Estuarine Studies, vol. 46. American Geophysical Union, Washington, DC, 373 pp.

Levitus, S., 1982. Climatological atlas of the world ocean. Technical report, NOAA Prof. Paper 13, US Government Printing Office, Washington, DC, $373 \mathrm{pp}$

Matthews, P., 1997. The impact of nonsynoptic sampling on mesoscale oceanographic surveys with towed instruments. J. Phys. Oceanogr. 14, 162-174.

McGillicuddy Jr., D.J., Lynch, D.R., Runge, J., Durbin, E.G., Gentleman, W.C., Davis, C.S., 2001. Evaluating the synopticity of the US GLOBEC Georges Bank broad-scale sampling pattern with observational system simulation experiments. Deep-Sea Res. II 48 (1/3), 483-499.

Nihoul, J.C.J., Deleersnijder, E., Djenidi, S., 1989. Modelling the general circulation of shelf seas by $3 \mathrm{D} k-\epsilon$ models. Earth-Sci. Rev. 26, 163-189.
Pedder, M., 1993. Interpolation and filtering of spatial observations using successive corrections and Gaussian filters. Mon. Weather Rev. 121, 2889-2902.

Perdersen, O.-P., Tande, K.S., Slagstad, D., 2000. A synoptic sampling method applied to Calanus finmarchicus population on the Norwegian mid-shelf in 1997. Mar. Ecol. Prog. Ser. 204, $143-$ 157.

Perkins, H., Kinder, T., La Violette, P., 1990. The Atlantic inflow in the Western Alboran Sea. J. Phys. Oceanogr. 20, 242-263.

Pinot, J.-M., Tintoré, J., Wang, D.-P., 1996. A study of the omega equation for diagnosing vertical motions at ocean fronts. J. Mar. Syst. 54, 239-259.

Pollard, R., Regier, L., 1992. Vorticity and vertical circulation at an ocean front. J. Phys. Oceanogr. 22, 609-625.

Rixen, M., Allen, J.T., Beckers, J.-M., 2001a. Diagnosing vertical velocities using the $\mathrm{QG}$ omega equation: a relocation method to obtain pseudo-synoptic data sets. Deep-Sea Res. 48 (6), $1347-$ 1373.

Rixen, M., Allen, J.T., Beckers, J.-M., 2001b. Non-synoptic versus pseudo-synoptic data sets: an assimilation experiment. Threedimensional ocean circulation: Lagrangian measurements and diagnostic. J. Mar. Syst. 29 (1-4), 313-333.

Rixen, M., Beckers, J.-M., Brankart, J.-M., Brasseur, P., 2001c. A numerically efficient data analysis method with error map generation. Ocean Modell. 2 (1-2), 45-60.

Sokolov, S., Rintoul, S., 1999. Some remarks on interpolation of nonstationary oceanographic fields. J. Atmos. Oceanic Technol. $16,1434-1449$.

Strass, V.H., 1994. Mesoscale instability and upwelling: Part 2. Testing the diagnostics of vertical motion with a three-dimensional ocean front model. J. Phys. Oceanogr. 24, 1759-1767.

Tchernia, P., 1977. Océanographie régionale. Description physique des océans et des mers. Ecole Nationale Supérieure de Techniques Avancées, Paris.

Tintoré, J., Damia, G., Alonso, S., 1991. Mesoscale dynamics and vertical motion in the Alboran Sea. J. Phys. Oceanogr. 21, $811-$ 823.

Viúdez, A., Tintoré, J., Haney, R., 1996. Circulation in the Alboran Sea as determined by quasi-synoptic hydrographic observations: Part I. Three-dimensional structure of the two anticyclonic gyres. J. Phys. Oceanogr. 26, 684-705.

Wahba, G., 1990. Spline Models for Observational Data. CBM-NSF Regional Conference Series in Applied Mathematics, vol. 59. Society for Industrial and Applied Mathematics, Philadelphia, PA, $169 \mathrm{pp}$.

Woods, J., 1988. In: Rothschild, B.J. (Ed.), Toward a Theory on Biological-Physical Interactions in the World Ocean. Kluwer, Dordrecht, pp. 7-38.

Wunsch, C., 1989. Sampling characteristics of satellite orbits. J. Atmos. Oceanic Technol. 6, 891-907. 